\title{
Association between oncological outcomes of patients with colorectal liver metastasis and additional gadoxetic acid-enhanced magnetic resonance imaging
}

\author{
Mizelle D’Silva^, Jai Young Cho, Ho-Seong Han, Yoo-Seok Yoon, Hae Won Lee, Jun Suh Lee, Boram Lee, \\ Moonhwan Kim \\ Department of Surgery, Seoul National University Bundang Hospital, Seoul National University College of Medicine, Seongnam-si, Gyeonggi-do, \\ Republic of Korea \\ Contributions: (I) Conception and design: JY Cho, HS Han; (II) Administrative support: YS Yoon, HW Lee; (III) Provision of study materials or \\ patients: JY Cho, HS Han, YS Yoon; (IV) Collection and assembly of data: M D'Silva, B Lee, M Kim; (V) Data analysis and interpretation: M D'Silva, \\ B Lee, JS Lee; (VI) Manuscript writing: All authors; (VII) Final approval of manuscript: All authors. \\ Correspondence to: Jai Young Cho, MD, PhD. Department of Surgery, Seoul National University Bundang Hospital, 82, Gumi-ro 173, Bundang-gu, \\ Seongnam-si, Gyeonggi-do, 13620, Republic of Korea. Email: jychogs@gmail.com.
}

Background: Gadoxetic acid-enhanced magnetic resonance imaging (MRI) has shown to be superior to
contrast-enhanced computed tomography (CT) in studies, there is no adequate data on its impact on overall
survival and recurrence-free survival in patients with colorectal liver metastasis. Our objective was to study
the benefit afforded by gadoxetic acid-enhanced MRI in patients with colorectal liver metastasis, especially in
terms of overall survival and recurrence-free survival.

Methods: Patients who underwent surgical treatment for colorectal liver metastasis between January 2003 and December 2018 were divided into groups depending on the pro-operative imaging carried out. Overall survival and recurrence-free survival were compared between both groups.

Results: Totally, 480 cases were included. Gadoxetic acid-enhanced MRI detected 220 new lesions in 123 cases. Presence of metastatic nodules was confirmed by gadoxetic acid-enhanced MRI in 33 of the 55 patients $(60.0 \%)$ with indeterminate nodules on contrast-enhanced CT. Gadoxetic acid-enhanced MRI detected significantly more nodules than contrast-enhanced CT (932 vs. 673, respectively, $\mathrm{P}<0.001$ ). There was no difference in the 5 -year overall survival $(\mathrm{P}=0.390)$ or in the 3 -month $(85.1 \% v s .86 .7 \%$, respectively, $\mathrm{P}=0.790)$, 6-month (78.0\% vs. 81.7\%, respectively, $\mathrm{P}=0.570)$, or 1 -year (65.7\% vs. 69.6\%, respectively, $\mathrm{P}=0.446)$ recurrence-free survival in patients examined with contrast-enhanced CT or gadoxetic acidenhanced MRI.

Conclusions: Although gadoxetic acid-enhanced MRI is more sensitive than contrast-enhanced CT, its utilization does not significantly affect the recurrence-free survival of the patient.

Keywords: Disappearing liver metastasis (DLM); indeterminate nodules; colorectal cancer (CRC); neoadjuvant chemotherapy; gadoxetic acid-enhanced magnetic resonance imaging (gadoxetic acid-enhanced MRI)

Submitted Jun 02, 2021. Accepted for publication Aug 09, 2021.

doi: 10.21037/apm-21-1470

View this article at: https://dx.doi.org/10.21037/apm-21-1470

$\wedge$ ORCID: 0000-0003-4434-2128. 


\section{Introduction}

Colorectal cancer (CRC) is the third most common cancer in the world (1). A recent study evaluated the global burden of CRC and has predicted that deaths due to CRC will increase substantially by 2035 (2). This being despite substantial improvements in the diagnostic modalities for metastatic CRC, superior chemotherapeutic and radiological agents, and better perioperative care over the last 20-30 years. The liver is the organ most frequently involved in CRC metastasis, and ablation or resection of liver lesions is the main treatment modality. Lesions that were previously considered unresectable can now be down staged with modern chemotherapeutic agents, thus becoming amenable to resection (3). With these clinical advances and the predicted increases in mortality looming, every effort should be made to precisely diagnose these metastatic lesions, so as to accurately stage the disease, and to treat it.

Computed tomography (CT) has been the primary investigation of choice for identifying colorectal liver metastasis (CRLM). Multidetector computed tomography (MDCT) has improved the sensitivity of diagnosis for these lesions by nearly $70-90 \%$. However, MDCT may be unable to characterize small lesions accurately, in which case magnetic resonance imaging (MRI) may be of value (4). Gadoxetic acidenhanced MRI (GE-MRI) better characterizes equivocal hepatic lesions, this advancement in radiology lead to the debate on the superiority between these tests. GE-MRI allows both static and dynamic hepatocyte imaging, which improves its accuracy of diagnosis. Although there is no consensus regarding any one technique, many studies have shown the results of GE-MRI to be superior to those of contrastenhanced CT (CECT). Therefore, we compared the value of MRI, particularly hepatocyte-specific MRI, and CECT in patients with CRLM. We present the following article in accordance with the STROBE reporting checklist (available at https://dx.doi.org/10.21037/apm-21-1470).

\section{Methods}

The study was conducted in accordance with the Declaration of Helsinki (as revised in 2013). This study was approved by the Institutional Review Board of Seoul National University Bundang Hospital, South Korea (IRB Approval Number B-2006/619/104) and individual consent for this retrospective analysis was waived. All patients with CRLM who underwent liver resection or intra-operative radio frequency ablation between January 2003 and December 2018 were analyzed retrospectively.

\section{Preoperative staging}

In the initial few years of the study period, CECT was used to diagnose CRLM. Later, with the availability of hepatocyte specific contrast, gadoxetic acid-enhanced liver MRI was performed in all patients with liver metastasis confirmed by CECT. Each lesion was classified with the consensus of two board-certified abdominal radiologists as one of three grades: definite metastasis, equivocal lesion, or definitive benign lesion. Hepatic lesions were classified as definitive metastases if they showed all of the following, rim enhancement in the arterial phase, a defect in the hepatobiliary phase, T2 hyperintensity, and diffusion restriction. Definitely benign hepatic lesions were defined as lesions with typical imaging findings, such as bright $\mathrm{T} 2$ signal intensity without enhancement for cysts and bright $\mathrm{T} 2$ signal intensity with a gradual centripetal filling-in pattern of enhancement for hemangiomas. The remaining hepatic lesions that showed neither the findings for metastatic lesions nor those for benign lesions were classified as "equivocal on MRI".

Surgical planning and decision-making were based on the results of CECT until 2008 and on GE-MRI results thereafter.

\section{CT image acquisition}

Contrast-enhanced helical CT scans of the abdomen and pelvis were obtained with a $\geq 16$-detector-row CT scanner (Brilliance $^{\circledR}$; Philips Healthcare, Cleveland, OH, USA). Patients were given $2 \mathrm{~mL}$ of iopromide (Ultravist $370^{\circledR}$; Schering, Berlin, Germany) per kilogram bodyweight, intravenously at a rate of $3 \mathrm{~mL} / \mathrm{s}$ via the antecubital vein. Imaging was performed in the portal venous phase with a scan delay of $60 \mathrm{~s}$ after the enhancement in the descending aorta reached $150 \mathrm{HU}$.

\section{MRI image acquisition}

Magnetic resonance images were obtained using 3.0T whole-body magnetic resonance systems (Philips HealthCare) with a 32-channel $(3.0 \mathrm{~T})$ phased-array coil as the receiver. Imaging used the dynamic contrast enhancement and conventional sequencing, including the dual-echo in- and opposed- phase spoiled gradient echo T1- 
weighted method; fat-saturated respiratory-triggered T2weighted method; and fat-saturated heavily T2-weighted and diffusion-weighted method (with b-factors of 0,50 , 500 , and $800 \mathrm{~s} / \mathrm{mm}^{2}$ ). Un-enhanced and contrast-enhanced (arterial, portal venous, 3-min delay, and hepatobiliary phases) transverse images were obtained using a T1weighted 3-dimensional gradient recalled echo sequence (T1 high-resolution isotropic volume examination, Philips HealthCare). Coronal images were also obtained for the hepatobiliary phase. Before imaging, intravenous gadoxetic acid was administrated via the antecubital vein using a power injector at a rate of $1 \mathrm{~mL} / \mathrm{s}$ for a dose of $0.025 \mathrm{mmol} / \mathrm{kg}$ of body weight, followed by a 20 -mL saline flush (5).

\section{Surgery}

The treatment plan for CRLM was decided based on the imaging and clinical findings of a multidisciplinary team, which consisted of colorectal surgeons, hepatobiliary surgeons, abdominal radiologists, pathologists, and radiation and medical oncologists. If a hepatic lesion remained equivocal even after GE-MRI, it was discussed by the team, who decided to closely observe it or evaluate it intraoperatively with ultrasonography or frozen biopsy. After the first surgery for CRC, the patients were followedup according to our postoperative surveillance protocol. Each patient underwent a physical examination and his/ her serum carcinoembryonic antigen (CEA) levels were measured every 3 months for 2 years, and then every 6 months for 5-8 years. CT scans of the chest, abdomen, and pelvis were performed every 3-6 months during the first 2 years, and then every 6-12 months thereafter. Positron emission tomography (PET) was selectively performed if needed, mainly in patients suspected of recurrence.

\section{Inclusion and exclusion criteria}

All patients were included, irrespective of whether they received neoadjuvant or adjuvant chemotherapy. Each new case even if underwent previous liver resection was considered a new entity. Prior adjuvant therapy in these cases was considered as neoadjuvant therapy. Synchronous (diagnosed simultaneously with the colorectal lesions) and metachronous lesions were included.

\section{Patient groups}

Patients who received neoadjuvant chemotherapy were first selected and those with disappearing liver metastases (DLMs) were followed-up and their outcomes noted.

Those who received adjuvant chemotherapy were then selected from the total 480 patients. They were divided into groups based on whether or not they underwent additional preoperative GE-MRI. The GE-MRI group included patients after 2008 who received adjuvant chemotherapy, while the no GE-MRI group included patients before 2008 who received adjuvant chemotherapy and some patients after 2008. Their clinico-pathological variables were assessed and the recurrence-free and overall survival were compared in the two groups.

\section{Statistical analysis}

All analyses were carried out using SPSS for Windows, version 20.0 (SPSS ${ }^{\circledR}$, Chicago, Illinois, USA). Categorical data were compared between groups using Pearson's $\chi^{2}$ test or Fisher's exact test. Continuous data were compared using Mann-Whitney U test. Kaplan-Meier survival curves were plotted to assess the disease-free survival and overall survival in the MRI and CT groups. Tests of statistical significance were two-tailed with $\mathrm{P}<0.05$ being considered as significant.

\section{Results}

\section{Interpretation of CT and GE-MRI}

A total of 480 cases were included in this study, 40 cases were patients who underwent repeat resections but were considered a new entity. One hundred and thirty/480 (27.1\%) cases received neoadjuvant chemotherapy and $369 / 480(76.9 \%)$ received adjuvant chemotherapy. GE-MRI detected 220 new lesions in 123 cases. Of the 55 patients with indeterminate nodules on CECT, GE-MRI confirmed the presence of metastatic nodules in $33 / 55(60.0 \%)$ of them. Patients who received neoadjuvant and adjuvant chemotherapy were selected and further analysis was done. The division of patients based on if they received adjuvant or neoadjuvant chemotherapy is shown in Figure 1.

\section{Neoadjuvant chemotherapy}

Neoadjuvant chemotherapy was administered to 130 patients, 48/130 (36.9\%) of whom had synchronous lesions. GE-MRI was performed in 110/130 (84.6\%) cases. Recurrence, either at the site of resection or in another part of the liver, was identified in 84/130 (64.6\%) cases. Disappearing lesions were 


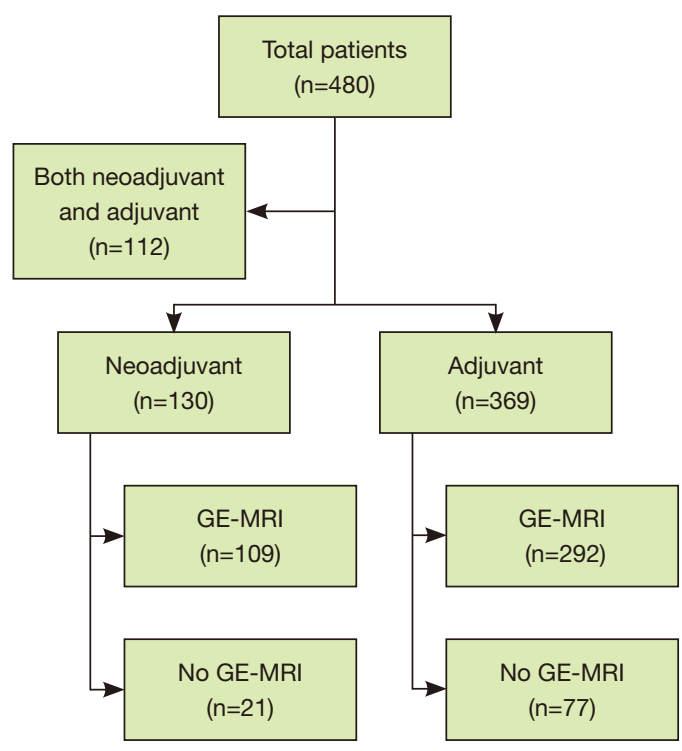

Figure 1 Flowchart indicating the different subgroups. GE-MRI, gadoxetic acid-enhanced magnetic resonance imaging.

present in $22 / 130(16.9 \%)$ cases, $15 / 22(68.2 \%)$ of whom experienced recurrence. The characteristics of cases with DLM are described in Table S1. Among the cases with DLM, male sex $(\mathrm{P}=0.020)$, those with lymphatic invasion in the primary $(\mathrm{P}=0.038)$ and lesions located in the posterosuperior segment $(\mathrm{P}=0.040)$ were associated with a higher incidence of recurrence. In a multivariate analysis, only lymphatic invasion $[\mathrm{P}=0.004,95 \%$ confidence interval (CI) 1.936-29.608] in the primary was an independent factor for recurrence (Table 1).

Ninety-eight/130 (75.4\%) cases had the same number of nodules despite receiving chemotherapy. No difference in recurrence-free survival was detected between the cases treated with CECT or GE-MRI at 1 year $(57.4 \%$ vs. $41.5 \%$, respectively, $\mathrm{P}=0.290)$, 3 years $(43.1 \%$ vs. $27.8 \%$, respectively, $\mathrm{P}=0.214)$, or 5 years $(28.7 \%$ vs. $39.5 \%$, respectively, $\mathrm{P}=0.415)$. Similarly, there was no difference in 5 -year overall survival in the two groups $(\mathrm{P}=0.305$; Figure 2$)$. Patients who received neoadjuvant chemotherapy showed no difference in recurrence-free survival or overall survival irrespective of whether a preoperative CECT or GE-MRI was performed.

\section{Adjuvant chemotherapy (Table 2)}

Adjuvant chemotherapy was administered to 369 (76.9\%) of the 480 cases. They were further classified into groups based on the use of GE-MRI. The GE-MRI group included
292/369 (79.1\%) cases and the no-GE-MRI group 77/369 (20.9\%) cases. The patients with GE-MRI more often received adjuvant chemotherapy. The clinicopathological data for both groups were similar, except that the number of anatomical resections $(\mathrm{P}=0.005)$ and the incidence of lymphatic invasion $(\mathrm{P}=0.033)$ were higher in the GE-MRI group. GE-MRI detected significantly more nodules than CECT (932 vs. 673, respectively, $\mathrm{P}<0.001$ ). However, even though the number of nodules detected were significantly greater, the use of GE-MRI did not affect the recurrencefree survival of the patients. There was no difference in the 5 -year overall survival rates $(\mathrm{P}=0.390)$ or in the 3 -month (85.1\% vs. $86.7 \%$, respectively, $\mathrm{P}=0.790)$, 6-month $(78.0 \%$ vs. $81.7 \%$, respectively, $\mathrm{P}=0.570)$, or $1-$ year $(65.7 \%$ vs. $69.6 \%$, respectively, $\mathrm{P}=0.446)$ disease-free survival rates in the patients examined with CECT or GE-MRI (Figures 3,4).

\section{Discussion}

In this study, GE-MRI was used to detect new lesions in 123 cases, and a total of 220 new lesions were detected. Kang et al. (5) showed that GE-MRI detected metastases in 90 patients who showed no lesions on CT, which caused the surgical plan to be altered in $3 \%$ of patients. There are two common types of hepatocyte-specific contrast medium, gadoxetate disodium (Eovist ${ }^{\circledR}$ or Primovist ${ }^{\circledR}$ Germany) and gadobenate dimeglumine (Multihance, Italy). Primovist ${ }^{\circledR}$ is the contrast agent used at our institute. After the injection of the contrast medium, it is distributed during two phases, the vascular and the extracellular phase. During and after the extracellular phase, a small amount is taken up by the hepatocytes and is finally cleared by the kidneys and biliary system. The optimal timing for contrast imaging is $20 \mathrm{~min}$ after contrast delivery (6). On T1-weighted MRI, the liver appears bright, whereas metastasis appears hypointense because there are no hepatocytes to take up the contrast agent. This enhancement of the liver parenchyma lasts up to $2 \mathrm{~h}$. The hepatocyte-specific nature of these contrast agents makes it easy to identify small lesions $(<1 \mathrm{~cm})$ accurately (7).

Although GE-MRI has the advantages of better detecting small lesions and better characterizing borderline lesions (dysplastic nodules and early hepatocellular carcinoma), it is not without limitations. Besides the drawbacks of high cost and patient claustrophobia, hepatocyte-specific MRI has further disadvantages of lower arterial and venous phase enhancement, a higher frequency of imaging artifacts during the arterial phase due to transient dyspnea and/or 
Table 1 Recurrence in patients with disappearing lesions

\begin{tabular}{|c|c|c|c|c|}
\hline Characteristic & Recurrence (n=15), n (\%) & No recurrence $(n=7), n(\%)$ & $P$ value & Multivariate analysis ( $\mathrm{P}$ value) \\
\hline Anatomical liver resection & $7(46.7)$ & $5(71.4)$ & 0.277 & - \\
\hline Posterior superior segment & $14(93.3)$ & $4(57.1)$ & 0.040 & 0.640 \\
\hline Bilobar tumour & $9(60.0)$ & $3(42.9)$ & 0.452 & - \\
\hline Perineural invasion in primary & $9(60.0)$ & $4(57.1)$ & 0.776 & - \\
\hline Venous invasion in primary & $8(53.3)$ & $4(57.1)$ & 0.577 & - \\
\hline Location of the primary & & & 0.141 & - \\
\hline Colon & $8(53.3)$ & $6(85.7)$ & & \\
\hline Adjuvant chemotherapy & $15(100.0)$ & $6(85.7)$ & 0.134 & - \\
\hline More than two lesions on MRI & $13(86.7)$ & $5(71.4)$ & 0.388 & - \\
\hline
\end{tabular}

MRI, magnetic resonance imaging.
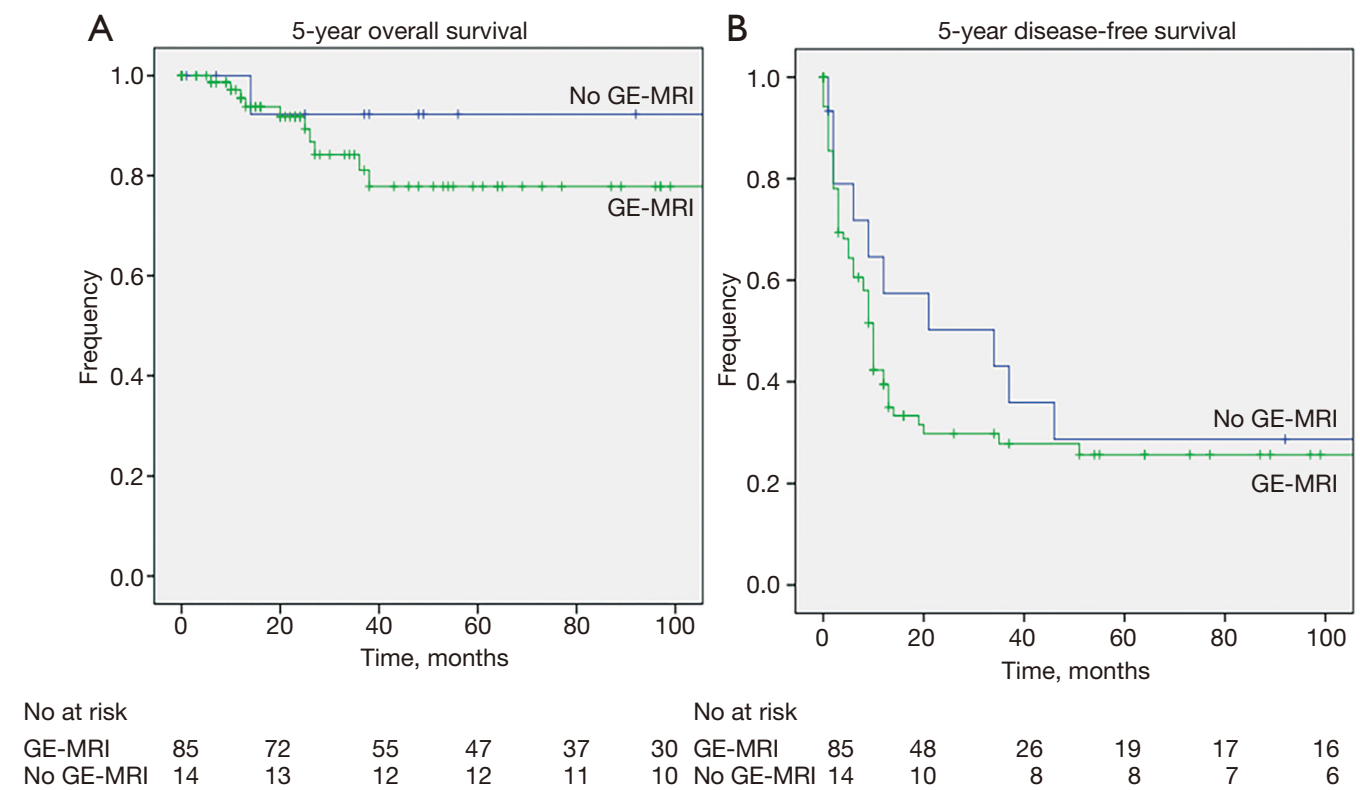

Figure 2 Five-year overall (A) and disease-free (B) survival curves for patients in whom the number of tumors was not affected by chemotherapy. GE-MRI, gadoxetic acid-enhanced magnetic resonance imaging.

severe transient motion, and the reduced uptake of contrast by the liver parenchyma in the presence of liver dysfunction and/or fibrosis (8).

Despite all these limitations, studies have demonstrated the superiority of GE-MRI over CECT in diagnosing CRLM (9). Asato et al. showed that GE-MRI has higher overall sensitivity than CECT $(91.4 \%$ vs. $80.9 \%$, respectively, $\mathrm{P}<0.001$ ), and also demonstrated its higher 
Table 2 Characteristics of patients who received adjuvant chemotherapy

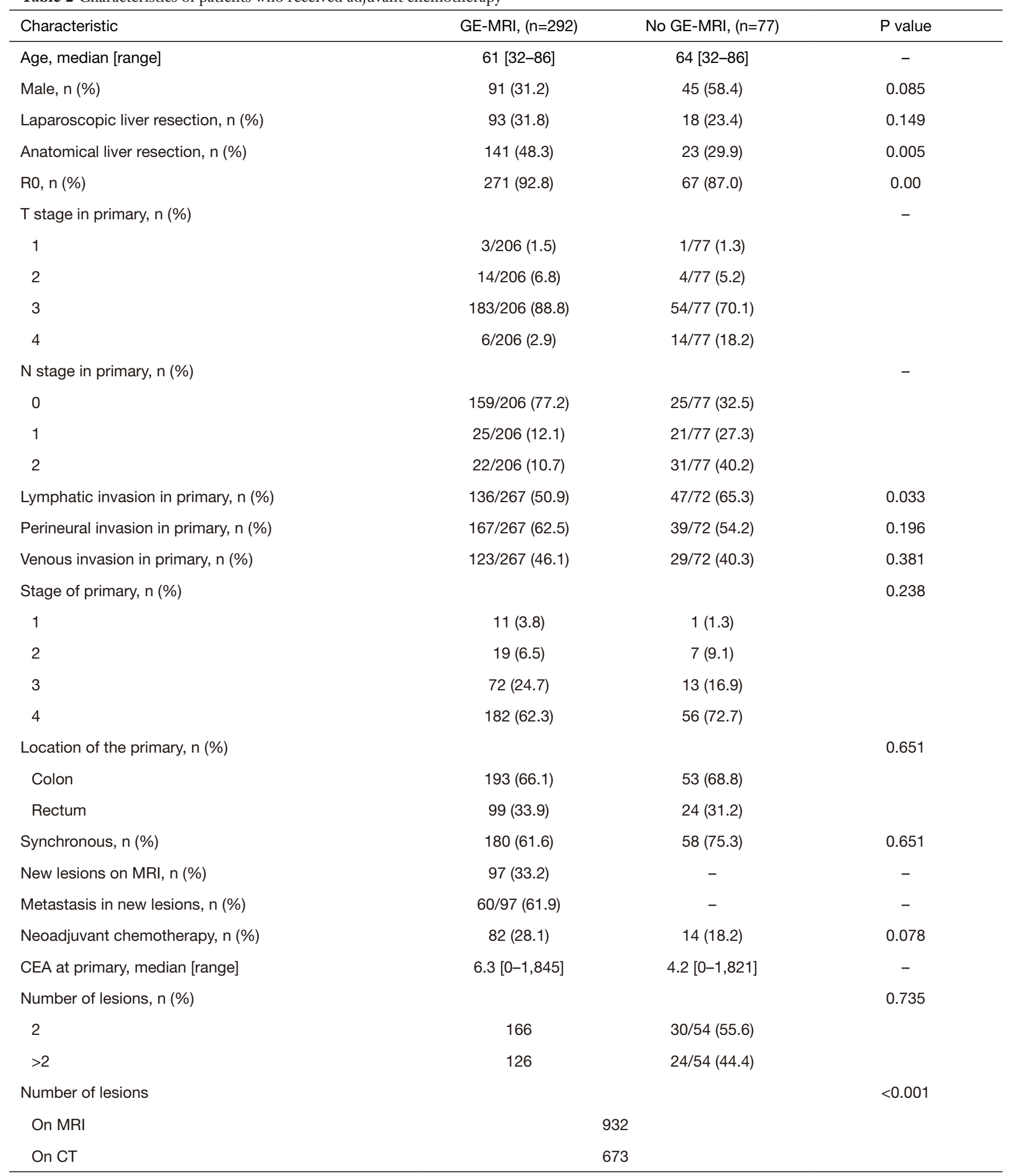

GE-MRI, gadoxetic acid-enhanced magnetic resonance imaging; T, tumor; N, nodal; CEA, carcinoembryonic antigen; CT, computed tomography. 

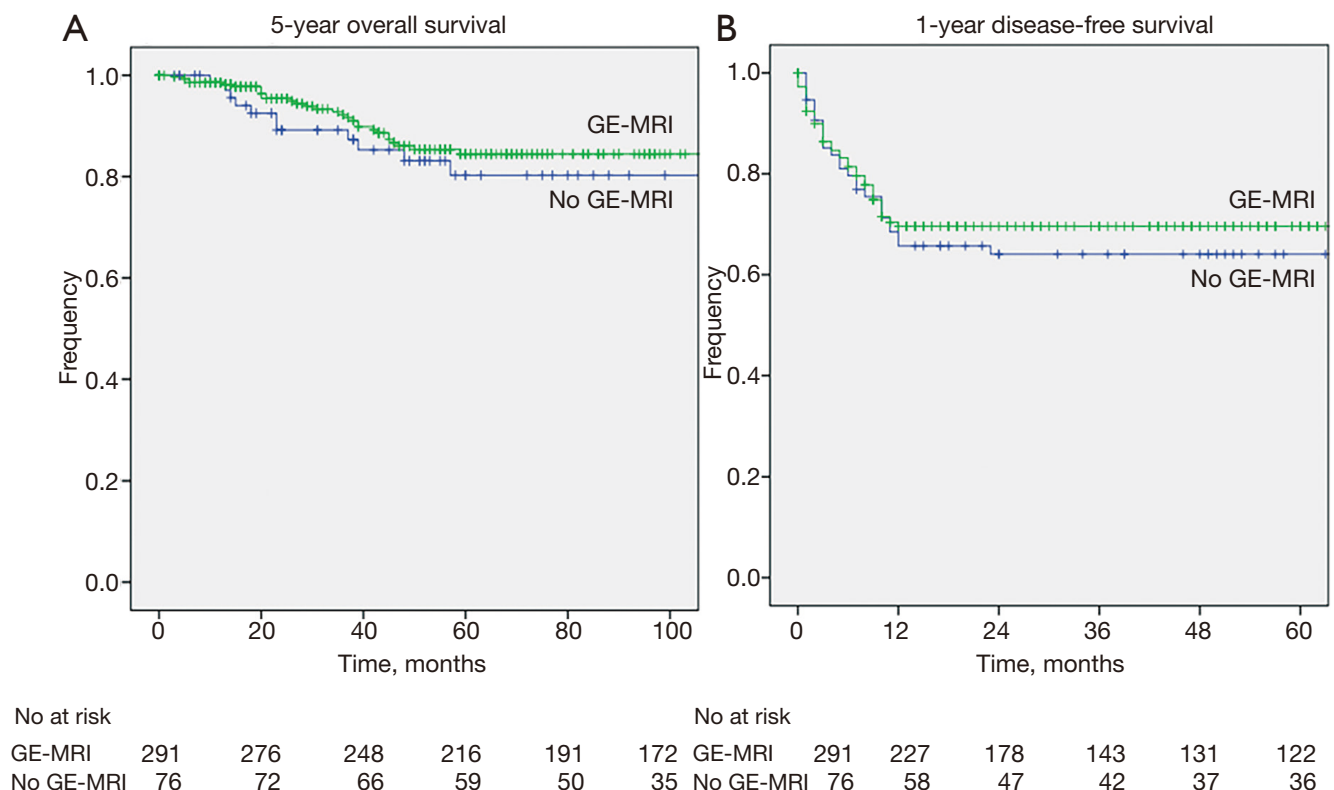

Figure 3 Five-year overall survival (A) and 1-year disease-free survival (B) curves for patients who received adjuvant chemotherapy. GE-MRI, gadoxetic acid-enhanced magnetic resonance imaging.

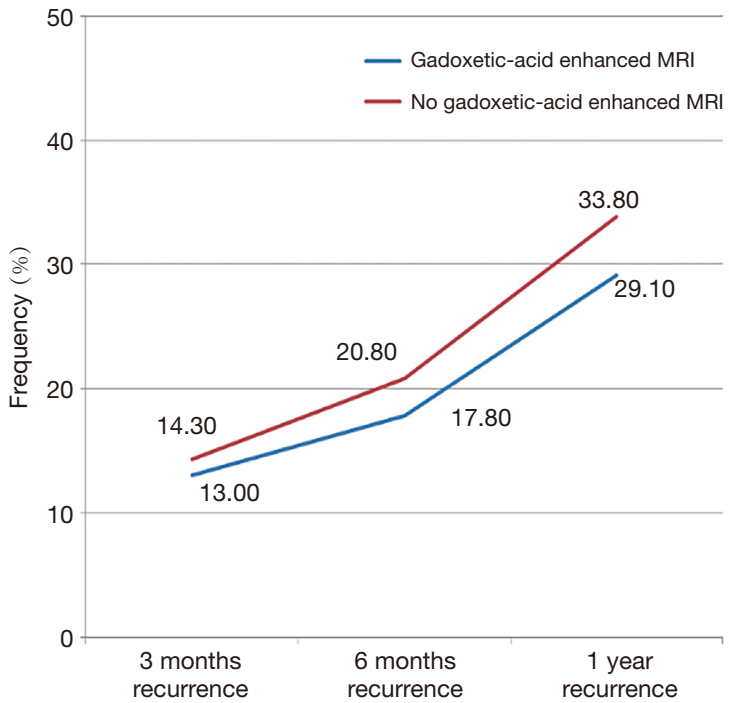

Figure 4 Cumulative recurrence over 1 year in patients who received adjuvant chemotherapy. MRI, magnetic resonance imaging.

sensitivity for smaller lesions (10). In a European study that compared the diagnostic efficacy of CECT, GE-MRI, and PET combined with CT, the per lesion sensitivity of GE-MRI was shown to be significantly higher than that of CECT or PET-CT (11). Similarly, a meta-analysis of 36 articles showed that GE-MRI had higher sensitivity than CECT and PET (12). CECT may be an inferior diagnostic technique after chemotherapy because the sinusoidal dilatation and injury caused by chemotherapeutic agents attenuate the hepatic parenchyma (13).

Neoadjuvant chemotherapy was administered to 130 (27.1\%) cases, after which GE-MRI identified 22 (16.9\%) cases with DLM. On follow-up, recurrence was detected in $15(68.2 \%)$ cases. Factors such as male sex, the posterior superior location of the tumor, and lymphatic invasion were associated with a higher incidence of recurrence in a univariate analysis. Lymphatic invasion from the primary lesion was the only independent factor for recurrence in DLM. A study by Tani et al. showed a recurrence rate of $33.3 \%$ in DLM, although when $70.3 \%$ of the initial DLMs were resected, $69.2 \%$ of those had residual cancer cells (14). Owen $e t a l$. reported similar results, with recurrence in $38.5 \%$ of DLMs and viable cancer cells in $55 \%$ of resected tumors (15).

We selected patients who received adjuvant chemotherapy and categorized them into two groups according to the use of GE-MRI. These patients were specifically selected to avoid bias because the addition of chemotherapy after surgery has been shown to reduce the recurrence rate of both liver metastasis and CRC itself, and to improve overall survival (16). All the clinicopathological data were similar 
in the two groups, except that the cases in the GE-MRI group underwent more anatomical resections and more cases in that group had significant lymphatic invasion from the primary tumor. GE-MRI detected significantly more nodules than CECT (932 vs. 673, respectively), consistent with all other studies that have compared GE-MRI with CECT and their diagnostic values (9). Although GE-MRI had a higher sensitivity for detecting nodules as compared to CECT, the recurrence rate postoperatively was not affected. This could be attributed to the fact that the size of the nodules being detected was not significant enough to cause a survival difference, besides some of the nodules identified on GE-MRI could be false positive. In addition, all of the detected nodules were not treated immediately, with some being under surveillance on follow-up. A study at Basingstoke between 2003 and 2009 studied the effect of GE-MRI before chemotherapy in patients with CRLM. It showed improved staging in $56 \%$ of patients and a reduced number of repeat hepatectomies. However, both groups had similar long-term survival rates (17).

Although GE-MRI is more sensitive than CT, the utilization of GE-MRI does not significantly affect the recurrence-free survival of the patient which raises the question of whether GE-MRI has any overall benefit for CRC patients.

This study had several limitations. It was a retrospective study with a small sample size. There were relatively few patients in the no-GE-MRI group because this technique was introduced at our center in 2008. The data is split into pre-2008 and post-2008. This temporal difference-on the range of a decade-is a confounding variable. Many other changes could have occurred in that decade which are hard to measure, generational changes in behavior, generation specific exposures, changes to ancillary care, technical advancements, change in post-surgery surveillance protocols, etc.

In conclusion, GE-MRI has many advantages over CECT, including improved rates of diagnosis and improved staging of tumors. Despite these advantages, recurrence-free survival and overall survival are not affected by the use of either modality. Therefore, when considering the advantages and disadvantages of GE-MRI, it remains unclear whether it should replace CECT for the detection of CRLM. GE-MRI could be used in patients with multiple nodules and those in whom the diagnosis of small equivocal lesions on CECT is difficult. However, the benefit actually afforded to each patient by GE-MRI must be evaluated.

\section{Acknowledgments}

Funding: None.

\section{Footnote}

Reporting Checklist: The authors have completed the STROBE reporting checklist. Available at https://dx.doi. org/10.21037/apm-21-1470

Data Sharing Statement: Available at https://dx.doi. org/10.21037/apm-21-1470

Peer Review File: Available at https://dx.doi.org/10.21037/ apm-21-1470

Conflicts of Interest: All authors have completed the ICMJE uniform disclosure form (available at https://dx.doi. org/10.21037/apm-21-1470). The authors have no conflicts of interest to declare.

Etbical Statement: The authors are accountable for all aspects of the work in ensuring that questions related to the accuracy or integrity of any part of the work are appropriately investigated and resolved. The study was conducted in accordance with the Declaration of Helsinki (as revised in 2013). This study was approved by Institutional Review Board at Seoul National University Bundang Hospital, South Korea (IRB Approval Number B-2006/619/104) and individual consent for this retrospective analysis was waived.

Open Access Statement: This is an Open Access article distributed in accordance with the Creative Commons Attribution-NonCommercial-NoDerivs 4.0 International License (CC BY-NC-ND 4.0), which permits the noncommercial replication and distribution of the article with the strict proviso that no changes or edits are made and the original work is properly cited (including links to both the formal publication through the relevant DOI and the license). See: https://creativecommons.org/licenses/by-nc-nd/4.0/.

\section{References}

1. Rawla P, Sunkara T, Barsouk A. Epidemiology of colorectal cancer: incidence, mortality, survival, and risk factors. Prz Gastroenterol 2019;14:89-103.

2. Araghi M, Soerjomataram I, Jenkins M, et al. Global 
trends in colorectal cancer mortality: projections to the year 2035. Int J Cancer 2019;144:2992-3000.

3. Chakedis J, Squires MH, Beal EW, et al. Update on current problems in colorectal liver metastasis. Curr Probl Surg 2017;54:554-602.

4. Cho JY, Lee YJ, Han HS, et al. Role of gadoxetic acidenhanced magnetic resonance imaging in the preoperative evaluation of small hepatic lesions in patients with colorectal cancer. World J Surg 2015;39:1161-6.

5. Kang SI, Kim DW, Cho JY, et al. Is MRI of the Liver Needed During Routine Preoperative Workup for Colorectal Cancer? Dis Colon Rectum 2017;60:936-44.

6. Trout AT, Towbin AJ, Smith EA, et al. Hepatocytespecific contrast media: not so simple. Pediatr Radiol 2018;48:1245-55.

7. Patel S, Cheek S, Osman H, et al. MRI with gadoxetate disodium for colorectal liver metastasis: is it the new "imaging modality of choice"? J Gastrointest Surg 2014;18:2130-5.

8. Fujita N, Nishie A, Asayama Y, et al. Hyperintense Liver Masses at Hepatobiliary Phase Gadoxetic Acid-enhanced MRI: Imaging Appearances and Clinical Importance. Radiographics 2020;40:72-94.

9. Jhaveri KS, Fischer SE, Hosseini-Nik H, et al. Prospective comparison of gadoxetic acid-enhanced liver MRI and contrast-enhanced CT with histopathological correlation for preoperative detection of colorectal liver metastases following chemotherapy and potential impact on surgical plan. HPB (Oxford) 2017;19:992-1000.

10. Asato N, Tsurusaki M, Sofue K, et al. Comparison of gadoxetic acid-enhanced dynamic MR imaging and contrast-enhanced computed tomography for preoperative evaluation of colorectal liver metastases. Jpn J Radiol 2017;35:197-205.

11. Sivesgaard K, Larsen LP, Sørensen M, et al. Diagnostic accuracy of CE-CT, MRI and FDG PET/CT for detecting colorectal cancer liver metastases in patients considered eligible for hepatic resection and/or local ablation. Eur Radiol 2018;28:4735-47.

12. Choi SH, Kim SY, Park SH, et al. Diagnostic performance of CT, gadoxetate disodium-enhanced MRI, and PET/CT for the diagnosis of colorectal liver metastasis: Systematic review and meta-analysis. J Magn Reson Imaging 2018;47:1237-50.

13. Lee DH, Lee JM, Hur BY, et al. Colorectal Cancer Liver Metastases: Diagnostic Performance and Prognostic Value of PET/MR Imaging. Radiology 2016;280:782-92.

14. Tani K, Shindoh J, Akamatsu N, et al. Management of disappearing lesions after chemotherapy for colorectal liver metastases: Relation between detectability and residual tumors. J Surg Oncol 2018;117:191-7.

15. Owen JW, Fowler KJ, Doyle MB, et al. Colorectal liver metastases: disappearing lesions in the era of Eovist hepatobiliary magnetic resonance imaging. HPB (Oxford) 2016;18:296-303.

16. Kobayashi S, Beppu T, Honda G, et al. Survival Benefit of and Indications for Adjuvant Chemotherapy for Resected Colorectal Liver Metastases-a Japanese Nationwide Survey. J Gastrointest Surg 2020;24:1244-60.

17. Knowles B, Welsh FK, Chandrakumaran K, et al. Detailed liver-specific imaging prior to pre-operative chemotherapy for colorectal liver metastases reduces intra-hepatic recurrence and the need for a repeat hepatectomy. HPB (Oxford) 2012;14:298-309.
Cite this article as: D'Silva M, Cho JY, Han HS, Yoon YS, Lee HW, Lee JS, Lee B, Kim M. Association between oncological outcomes of patients with colorectal liver metastasis and additional gadoxetic acid-enhanced magnetic resonance imaging. Ann Palliat Med 2021;10(10):10213-10221. doi: /10.21037/ apm-21-1470 


\section{Supplementary}

Table S1 Characteristics of patients with disappearing liver metastases

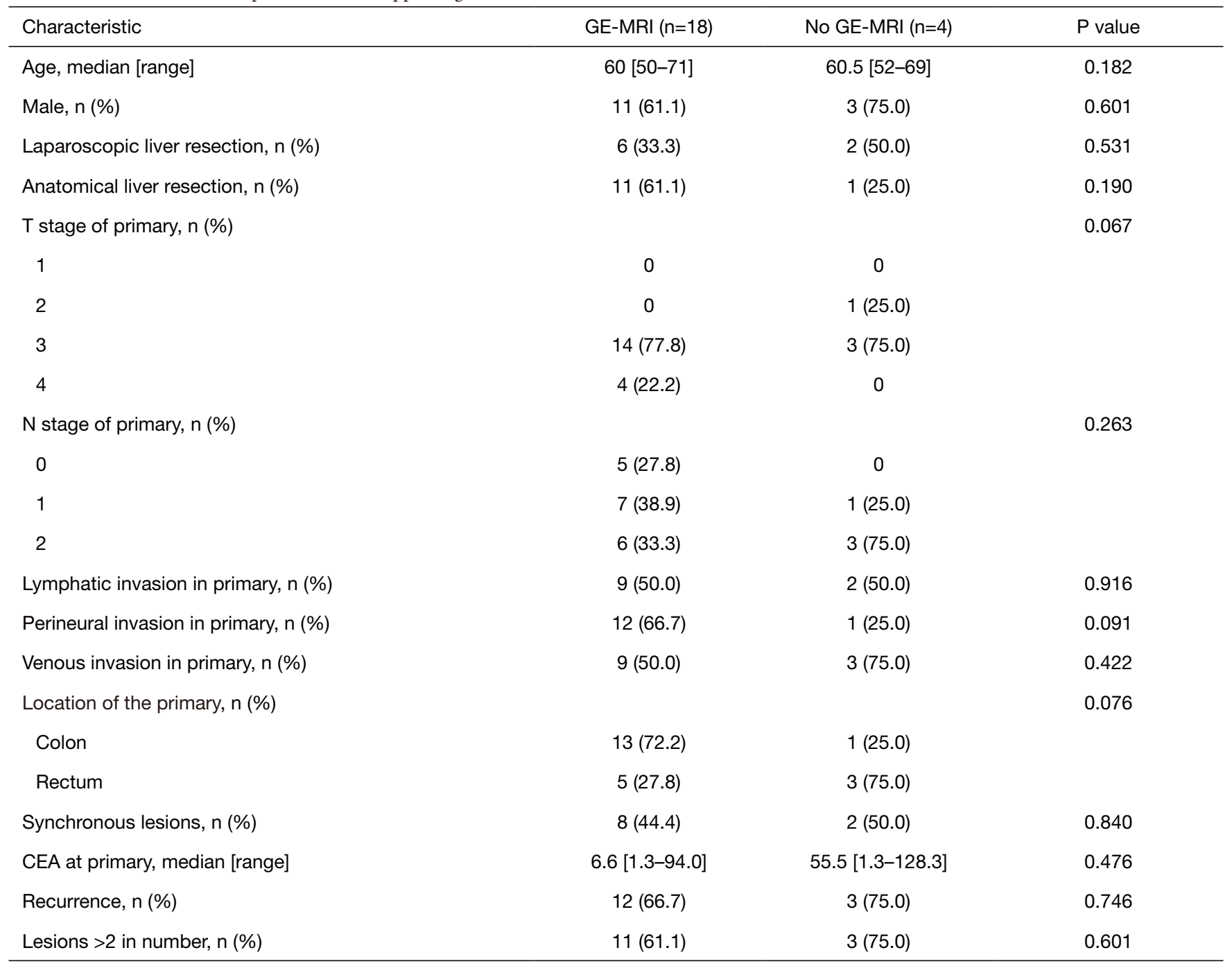

GE-MRI, gadoxetic acid-enhanced magnetic resonance imaging; T, tumor; N, nodal; CEA, carcinoembryonic antigen; CT, computed tomography. 